\title{
STRENGTH TRAINING PROMOTES ANTHROPOMETRIC AND FUNCTIONAL BENEFITS IN SEDENTARY SUBJECTS: DOES A PERSONAL TRAINER MATTER?
}

original paper

( ) University School of Physical Education in Wroclaw

DOI: https://doi.org/10.5114/hm.2021.103286

\section{ALINE RODRIGUES MATOS ${ }^{1}$, HEITOR SIQUEIRA RIBEIRO ${ }^{2,3}$, HUGO DE LUCA CORRÊA ${ }^{1}$, APARECIDO PIMENTEL FERREIRA ${ }^{3,4}$, ELAINE VIEIRA ${ }^{1}$}

${ }^{1}$ Postgraduate Program on Physical Education, Catholic University of Brasília, Federal District, Brazil

${ }^{2}$ Faculty of Physical Education, University of Brasília, Brasília, Brazil

${ }^{3}$ Interdisciplinary Research Department, University Centre ICESP, Brasília, Brazil

${ }^{4}$ Santa Úrsula University, Rio de Janeiro, Brazil

\section{ABSTRACT}

Purpose. The purpose of this study was to compare the effects of strength training performed with and without the supervision of a personal trainer on the anthropometric, functional, and biochemical responses of sedentary adults.

Methods. Overall, 38 sedentary men were divided into 3 groups: control group $(n=12)$, no personal trainer group $(n=14)$, and personal trainer group $(n=12)$. Participants of all groups were submitted to pre-training data collection, including anthropometric evaluation, functional and cardiometabolic tests, and blood collection for biochemical parameters. Then, the subjects were involved in strength training of 50 minutes 3 times per week for 8 weeks. After the strength training program, the participants were submitted to the same data collection.

Results. The personal trainer group presented a decrease in waist circumference, systolic blood pressure, sum of skinfolds, abdominal resistance, pectoral resistance, one-repetition maximum on bench and leg press compared with the no personal trainer group $(p<0.05)$. The no personal trainer group had an improvement only in the sum of skinfolds $(p<0.05)$.

Conclusions. The results of the present study indicate that 8 weeks of strength training with a personal trainer can produce important changes in body composition and blood pressure in sedentary subjects.

Key words: supervised training, strength exercise, blood pressure, sedentary lifestyle

\section{Introduction}

Insufficient physical activity is a leading risk factor for metabolic diseases and has a negative impact on the quality of life. The World Health Organization recognizes a strong association between physical activity and major non-communicable diseases and agreed to a $10 \%$ relative reduction in the prevalence of insufficient physical activity by 2025 . However, a recent report including data from 358 surveys across 168 countries, including 1.9 million participants, concluded a global age-standardized prevalence of $27.5 \%$ of insufficient physical activity. Thus, the global target of a $10 \%$ relative reduction of insufficient physical activity by 2025 will not be met unless accelerated policies to increase population levels of physical activity are implemented [1].

In the context of this problematic scenario, the practice of physical exercise and lifestyle changes should be encouraged owing to the important and efficient physiologic adaptations and mental health benefits as a non-pharmacological tool in the prevention of noncommunicable diseases [2,3]. Among the various modalities of physical exercise, strength training (ST) plays an important role, since the physiological changes provided by ST have been demonstrated to be efficient to prevent metabolic diseases and obesity $[4,5]$. Moreover, some studies show that the strength-enhancing effect is only achieved when threshold intensity is consistently targeted [6, 7]. The use of self-selected inten-

Correspondence address: Elaine Vieira, Postgraduate Program on Physical Education, Universidade Católica de Brasília, Campus I - QS 07 - Lote 01 - EPCT - Águas Claras - Brasília, DF, CEP: 71966-700 Brazil, e-mail: elaine.vieira@ucb.br

Received: December 3, 2019

Accepted for publication: May 22, 2020

Citation: Matos AR, Ribeiro HS, Corrêa HL, Ferreira AP, Vieira E. Strength training promotes anthropometric and functional benefits in sedentary subjects: does a personal trainer matter? Hum Mov. 2021;22(4):20-27; doi: https://doi.org/10.5114/ hm.2021.103286. 
sity training leads to intensities that are below the stipulated limits to promote relevant physiological adaptations [8-10]. Therefore, a personal trainer seems to be essential in the achievement of a threshold intensity which could cause a better response to ST, due to correcting exercise techniques and controlling the training intensity, volume, frequency, and structure [9-11]. Additionally, a personal trainer also plays a role in reinforcing progression toward goal attainment by providing psychological stimuli (including elaboration of the training protocol, social contact during training, instruction, and correcting exercise techniques), which increase motivation [11].

The effects of training with a personal trainer in the improvement of the anthropometric and functional profile are well known [9-11]; however, up to the present moment, no studies have verified its connection with the biochemical profile (i.e., lipid profile, blood glucose, and insulin). We hypothesized that a personal trainer would be very important in the control of the exercise intensity that could cause a better physiological response to ST. This strict intensity control could change the anthropometric, functional, and biochemical profile of the participants.

Therefore, to fill the gap related to the effect of training with a personal trainer on anthropometric, functional, and biochemical variables, the present study aims to compare the impact of ST performed with and without the supervision of a personal trainer on the anthropometric, functional, and biochemical responses in sedentary adults.

\section{Material and methods}

\section{Subjects}

A total of 38 sedentary men aged 20-40 years volunteered to participate in this study and were informed about the procedures of the experiment and its implications. They were divided into 3 groups: control group (CG, $n=12$; age: $28.5 \pm 7.1$ years), no personal trainer group (NPTG, $n=14$; age: $36.2 \pm 5.5$ years, $p=0.022$ vs. control), and personal trainer group (PTG, $n=12$; age: $32.4 \pm 7.5$ years). The inclusion criteria were the following: good physical condition and physician's consent to perform ST, participation in all initial tests, and completing a minimum of $75 \%$ of the training sessions. The volunteers of all groups were submitted to pre-training examination, which included anthropometric evaluation, functional and cardiometabolic tests, and blood collection for biochemical parameters. Then, they were involved in ST of $50 \mathrm{~min}-$ utes 3 times per week for 8 weeks. After the 8 weeks of ST, the subjects were submitted to post-training data collection.

\section{Strength training program}

Both NPTG and PTG performed similar training, differing only in the constant monitoring of a personal trainer. The PTG had constant monitoring in each exercise session, and the NPTG received instructions at the beginning of the training and every 3 weeks. The ST program was conducted as described below: 1 week for familiarization and learning of movements; 3 weeks with training $A ; 2$ weeks with training $B$; 2 weeks with training $C$. Training A was used to learn the movements; however, the load would be adjusted to perform 2 sets of 15 repetitions with the subjective rate of perceived exertion between 0 and 10 . The numbers of repetitions of each series were maximal and equalled approximately 12 repetitions in training $\mathrm{A}$ and 8 repetitions in workouts $B$ and $C$ (Table 1 ). The cadence described in Table 1 refers to the movement speed, where the first number means the concentric contraction, the second one is the transition to the eccentric contraction, the third one represents the eccentric contraction itself, and the fourth number refers to the transition from eccentric to concentric contractions.

\section{Anthropometric assessments}

Body mass, height, and skinfolds were determined in accordance with the procedures described before [12]. For body height and mass, scales and a stadiometer (FilizolaTM model PL - 200, Beyond Technology, São Paulo, Brazil) were used with a resolution of $0.1 \mathrm{~kg}$ and $0.1 \mathrm{~cm}$. The skinfolds were measured with an adipometer (Lange, Beta Technology Incorporated, Cambridge, MD, USA) with a resolution of $1 \mathrm{~mm}$; the data were collected in triplicate by the same evaluator and always on the right side of the body. For body density estimation, we used the equation by Jackson and Pollock [13].

\section{Flexibility test}

The subject sits on a rubber matting with knees straight, legs separated just enough to straddle the stern board of the scale platform, with the feet placed in the footprints on the cross board and pressed firmly against the board. The arms are extended forward with the hands placed palms down on the upper surface of the scale. In this position, the subject bobs for- 


\section{HUMAN MOVEMENT}

A. Matos et al., The importance of a personal trainer in sedentary subjects

Table 1. Exercise training prescription and periodization

\begin{tabular}{lccccc}
\hline Exercise & Series & Intensity & Repetitions & Cadence & Rest \\
\hline Bench press & \multicolumn{3}{c}{ TRAINING A } & & \\
Incline bench press & 3 & $70 \%$ & Up to 12 & 2020 & 45 seconds \\
Pulldown & 2 & $70 \%$ & Up to 12 & 2020 & 45 seconds \\
Low row & 3 & $70 \%$ & Up to 12 & 2020 & 45 seconds \\
Squat & 2 & $70 \%$ & Up to 12 & 2020 & 45 seconds \\
Seated leg extension & 3 & $70 \%$ & Up to 12 & 2020 & 45 seconds \\
Seated leg curl & 3 & $70 \%$ & Up to 12 & 2020 & 45 seconds \\
Crunch abdominal & 3 & $70 \%$ & Up to 12 & 2020 & 45 seconds \\
\hline
\end{tabular}

\begin{tabular}{|c|c|c|c|c|c|}
\hline Warm-up & \multicolumn{5}{|c|}{10 minutes on cycle ergometer or treadmill } \\
\hline Stretching & \multicolumn{5}{|c|}{ Static stretching 20 seconds per position of major muscle groups } \\
\hline \multicolumn{6}{|c|}{ TRAINING B } \\
\hline Bench press & 4 & $80 \%$ & Up to 8 & 2020 & 60 seconds \\
\hline Incline dumbbell fly & 2 & $80 \%$ & Up to 8 & 2020 & 60 seconds \\
\hline Lat pulldown & 4 & $80 \%$ & Up to 8 & 2020 & 60 seconds \\
\hline Low row & 2 & $80 \%$ & Up to 8 & 2020 & 60 seconds \\
\hline Squat & 4 & $80 \%$ & Up to 8 & 2020 & 60 seconds \\
\hline Seated leg extension & 3 & $80 \%$ & Up to 8 & 2020 & 60 seconds \\
\hline Stiff & 3 & $80 \%$ & Up to 8 & 2020 & 60 seconds \\
\hline Crunch abdominal & 3 & & 30 & & 60 seconds \\
\hline
\end{tabular}

\begin{tabular}{|c|c|c|c|c|c|}
\hline Warm-up & \multicolumn{5}{|c|}{10 minutes on cycle ergometer or treadmill } \\
\hline Stretching & \multicolumn{5}{|c|}{ Static stretching 20 seconds per position of major muscle groups } \\
\hline \multicolumn{6}{|c|}{ TRAINING C } \\
\hline Decline dumbbell bench press & 4 & $80 \%$ & Up to 8 & 2020 & 60 seconds \\
\hline Pulldown & 4 & $80 \%$ & Up to 8 & 2020 & 60 seconds \\
\hline Shoulder lift & 4 & $80 \%$ & Up to 8 & 2020 & 60 seconds \\
\hline Shoulder press machine & 4 & $80 \%$ & Up to 8 & 2020 & 60 seconds \\
\hline Lunge & 3 & $80 \%$ & Up to 8 & 2020 & 60 seconds \\
\hline Leg press $45^{\circ}$ & 4 & $80 \%$ & Up to 8 & 2020 & 60 seconds \\
\hline Stiff deadlift & 3 & $80 \%$ & Up to 8 & 2020 & 60 seconds \\
\hline Crunch abdominal & 3 & & 30 & & 60 seconds \\
\hline
\end{tabular}

Warm-up

10 minutes on cycle ergometer or treadmill

Stretching

Static stretching 20 seconds per position of major muscle groups

ward 4 times and holds the position of maximum reach on the fourth count. The score is the most distant point reached and held on the fourth movement [14].

Muscle endurance test 1 minute abdominal

We performed the tests of muscular resistance of 1-minute abdominal crunches, lying on the back and place as soles of the feet in full contact with the ground, the knees flexed, and the heels about $35 \mathrm{~cm}$ away from the buttocks; crossing arms over chest, with hands resting on shoulders on opposite sides. The subject puts the chin against the chest, 'curls' the body until reaching a position sent; when the elbows touch the thigh, a repetition counts. Using a stopwatch or clock with a seconds hand, the evaluator started and counted the number of sit-ups in a minute [15].

\section{Muscle endurance test located \\ 1-minute arm push-up}

We performed the tests of muscular resistance of 1-minute arm push-up. The 1-minute arm flexion followed the protocol published by Mayhew et al. [16]. 


\section{Intermittent recovery test - level 1}

The test aimed to indirectly evaluate $\mathrm{VO}_{2} \max$. It was performed in a flat location with 2 marks having a 20-m distance straight between them, with a rest area of $5 \mathrm{~m}$ on the initial side of the test. At the end of the test, the total distance travelled was recorded to later calculate $\mathrm{VO}_{2}$ max using the following formula (where IR stands for intermittent recovery) [17]:

$$
\begin{aligned}
\mathrm{VO}_{2} \max (\mathrm{ml} / \mathrm{min} / \mathrm{kg}) & =\text { distance IR1 }(\mathrm{m}) \times 0.0084 \\
& +36.4
\end{aligned}
$$

\section{Analysis of biochemical parameters}

Blood was collected in the pre- and post-training periods. The following biochemical parameters were analysed: blood glucose levels, plasma insulin, total cholesterol, triglycerides, high-density lipoprotein (HDL), low-density lipoprotein (LDL), very low-density lipoprotein (VLDL), and total lipids. Blood glucose was measured with a DOLES kit (Goiânia, Brazil, Biosystems A15). Plasma insulin was determined by CalSet (EUA; Elecsys Insulin CalSet), and lipids were evaluated with a Biosystems kit (Costa Branca, Barcelona, Spain).

\section{Cold pressure test}

The cold pressure test was used with a styrofoam box and a thermometer capable of measuring the maximum value of $70^{\circ} \mathrm{C}$ and the minimum value of $-50^{\circ} \mathrm{C}$. Blood pressure and heart rate were determined before and after the individual dipped the right hand up to the height of the wrist inside the box with ice water, with a temperature of $4-5^{\circ}$, for 1 minute, withdrew the hand, and remained seated.

\section{Analysis of cardiometabolic parameters}

The analysis of the cardiometabolic parameters was performed by means of blood pressure measurement with a digital device (Microlife ${ }^{\circledR}$, model BP 3AC1-1, Swit- zerland) and pre- and post-training heart rate measurement by using a Polar cardiac monitor (S810i, USA).

\section{Statistics design}

Data were expressed as mean and standard deviation. Normality was tested with the Shapiro-Wilk and Levene's test. A variance analysis (one way ANOVA) with a Tukey's post-hoc test was performed to compare the deltas (post-pre) between the CG, NPTG, and PTG. The paired $t$-test was applied in all groups to identify possible differences between the pre- and post-training period. Statistical significance of the results was accepted at $p<0.05$. The program used was SPSS version 2.2 .

\section{Ethical approval}

The research related to human use has complied with all the relevant national regulations and institutional policies, has followed the tenets of the Declaration of Helsinki, and has been approved by the Research Ethics Committee of the Catholic University of Brasília, Brazil.

\section{Informed consent}

Informed consent has been obtained from all individuals included in this study.

\section{Results}

The variables referring to the intensity and total volume in the protocol are described in Table 2, presenting the mean heart rate, frequency, workload, and rate of perceived exertion. There was no difference between groups in any training variable $(p>0.05)$; thus, each group had the same total workload after the intervention period (Table 2).

Table 3 presents the comparison between pre- and post-training follow-up for anthropometric, functional, haemodynamic, and clinical variables in each group. The PTG exhibited a decrease in waist circumference, systolic blood pressure, sum of skinfolds, abdominal resistance, pectoral resistance, one-repetition maxi-

Table 2. Variables referring to the intensity and total volume in the protocol

\begin{tabular}{lcccc}
\hline Parameters & Total & NPTG & PTG & $p$ \\
\hline Heart rate (bpm) & $124.2 \pm 11.2$ & $123.5 \pm 8.4$ & $125 \pm 14.1$ & 0.751 \\
Frequency (days) & $23.5 \pm 0.7$ & $23.4 \pm 0.8$ & $23.5 \pm 0.5$ & 0.588 \\
Volume (repetitions) & $298 \pm 7.3$ & $299.2 \pm 3.3$ & $296.5 \pm 10.1$ & 0.362 \\
RPE & $8.1 \pm 0.7$ & $8 \pm 0.7$ & $8.2 \pm 0.6$ & 0.505 \\
\hline
\end{tabular}

NPTG - no personal trainer group, PTG - personal trainer group, RPE - rate of perceived exertion 


\section{HUMAN MOVEMENT}

A. Matos et al., The importance of a personal trainer in sedentary subjects

Table 3. Anthropometric, haemodynamic, functional, and biochemical response to 8 weeks of strength training

\begin{tabular}{|c|c|c|c|c|c|c|}
\hline \multirow[b]{2}{*}{ Parameters } & \multicolumn{2}{|c|}{ CG } & \multicolumn{2}{|c|}{ NPTG } & \multicolumn{2}{|c|}{ PTG } \\
\hline & $\begin{array}{l}\text { Before } \\
\text { training }\end{array}$ & $\begin{array}{c}\text { After } \\
\text { training }\end{array}$ & $\begin{array}{l}\text { Before } \\
\text { training }\end{array}$ & $\begin{array}{c}\text { After } \\
\text { training }\end{array}$ & $\begin{array}{l}\text { Before } \\
\text { training }\end{array}$ & $\begin{array}{c}\text { After } \\
\text { training }\end{array}$ \\
\hline Body mass (kg) & $95.4 \pm 19.7$ & $97.1 \pm 17.8$ & $97.1 \pm 21.0$ & $96.3 \pm 18.4$ & $98.9 \pm 23.3$ & $97.2 \pm 21.8$ \\
\hline Body mass index $\left(\mathrm{kg} / \mathrm{m}^{2}\right)$ & $31.3 \pm 6.2$ & $31.5 \pm 5.5$ & $32.3 \pm 5.3$ & $32.1 \pm 4.7$ & $32.1 \pm 6.5$ & $31.6 \pm 6.2$ \\
\hline Waist circumference $(\mathrm{cm})$ & $99.8 \pm 13.3$ & $98.6 \pm 11.3$ & $100.3 \pm 14.3$ & $97.8 \pm 10.7$ & $101.7 \pm 15.2$ & $98.2 \pm 12.7^{*}$ \\
\hline Heart rate $(\mathrm{bpm})$ & $79 \pm 15.5$ & $80 \pm 20.8$ & $83.1 \pm 9.3$ & $78.5 \pm 9.5$ & $83 \pm 10.1$ & $80.3 \pm 9.3$ \\
\hline Systolic blood pressure (mm Hg) & $110.6 \pm 43.4$ & $130.6 \pm 22.3$ & $127.9 \pm 6.9$ & $122.9 \pm 12.3$ & $132.8 \pm 16.5$ & $121.9 \pm 6.0^{*}$ \\
\hline Diastolic blood pressure (mm Hg) & $75.8 \pm 8.2$ & $81.4 \pm 13.5$ & $81.7 \pm 9.2$ & $77.7 \pm 7.8$ & $78.6 \pm 8.7$ & $74.5 \pm 6.8$ \\
\hline$\Sigma$ skinfold & $203.7 \pm 48.5$ & $193.5 \pm 40.2$ & $206.8 \pm 48.3$ & $193.7 \pm 45.5^{*}$ & $212.2 \pm 53.5$ & $196.1 \pm 42.9 *$ \\
\hline $\mathrm{VO}_{2} \max (\mathrm{ml} / \mathrm{kg} / \mathrm{min})$ & $38.9 \pm 0.9$ & $39.1 \pm 1.3$ & $38.6 \pm 0.8$ & $38.8 \pm 0.8$ & $38.7 \pm 0.7$ & $38.9 \pm 1.0$ \\
\hline Flexibility $(\mathrm{cm})$ & $17.5 \pm 6.5$ & $17.5 \pm 6.5$ & $18.1 \pm 9.0$ & $19.2 \pm 7.2$ & $19.3 \pm 11.4$ & $21.5 \pm 11.8^{*}$ \\
\hline Abdominal resistance (repetitions) & $22.4 \pm 9.2$ & $24.5 \pm 8.6$ & $21.8 \pm 9.4$ & $25.8 \pm 8.6$ & $22.1 \pm 10.8$ & $27.5 \pm 11.0^{*}$ \\
\hline Pectoral resistance (repetitions) & $17.0 \pm 4.7$ & $17.3 \pm 5.9$ & $18 \pm 7.4$ & $20.5 \pm 6.8$ & $15.9 \pm 6.0$ & $22.3 \pm 7.6^{*}$ \\
\hline Leg press $1 \mathrm{RM}(\mathrm{kg})$ & $231.6 \pm 79.7$ & $308.3 \pm 88.3^{*}$ & $286.4 \pm 40.1$ & $375.3 \pm 64.8$ & $250.8 \pm 41.6$ & $351.6 \pm 85.0 *$ \\
\hline Bench press 1RM (kg) & $57 \pm 19.1$ & $66.3 \pm 17.3^{*}$ & $61.8 \pm 8.3$ & $70 \pm 14.1$ & $55.8 \pm 14.8$ & $64.1 \pm 17.8^{*}$ \\
\hline Blood glucose (mg/dl) & $101.2 \pm 8.8$ & $100.6 \pm 4.9$ & $103 \pm 10.2$ & $106.7 \pm 17.1$ & $100.9 \pm 6.3$ & $98.7 \pm 7.7$ \\
\hline Insulin (mg/dl) & $10.5 \pm 6.6$ & $12.6 \pm 6.6$ & $5.6 \pm 4.1$ & $9.1 \pm 5.8$ & $10.1 \pm 7.3$ & $10.7 \pm 5.3$ \\
\hline Cholesterol (mg/dl) & $221.6 \pm 58.6$ & $195.4 \pm 43.0^{*}$ & $191 \pm 55.7$ & $199.6 \pm 45.6$ & $192.4 \pm 41.8$ & $191.2 \pm 27.7$ \\
\hline Triglycerides (mg/dl) & $187.3 \pm 167.9$ & $151.5 \pm 108.7$ & $220.8 \pm 205.7$ & $205.2 \pm 174.9$ & $229.3 \pm 201.5$ & $189.5 \pm 150.7$ \\
\hline HDL (mg/dl) & $33.3 \pm 9.1$ & $55.3 \pm 62.0$ & $37.2 \pm 10.8$ & $38.8 \pm 8.7$ & $34.9 \pm 7.2$ & $34.4 \pm 5.0$ \\
\hline LDL (mg/dl) & $154.3 \pm 59.9$ & $128.4 \pm 52.2$ & $116.2 \pm 41.4$ & $123.9 \pm 42.7$ & $125.1 \pm 35.9$ & $122.3 \pm 16.9$ \\
\hline VLDL (mg/dl) & $31.0 \pm 26.5$ & $24.4 \pm 8.5$ & $26.7 \pm 18.8$ & $25.4 \pm 18.0$ & $29.8 \pm 12.5$ & $25.4 \pm 8.4$ \\
\hline Total lipids (mg/dl) & $710.2 \pm 213.4$ & $623 \pm 124.0$ & $559.7 \pm 182.6$ & $587.5 \pm 177.2$ & $608.5 \pm 140.1$ & $596.3 \pm 64.9$ \\
\hline
\end{tabular}

* significant difference as compared with the pre-training period $(p<0.05)$

CG - control group, NPTG - no personal trainer group, PTG - personal trainer group, 1RM - one-repetition maximum,

HDL - high-density lipoprotein, LDL - low-density lipoprotein, VLDL - very low-density lipoprotein

Table 4. Comparison of the effects of resistance training between groups

\begin{tabular}{lcccc}
\hline & & Delta & PTG & Total \\
\cline { 2 - 4 } Parameters & CG & NPTG & $-0.7 \pm 4.3$ & $-0.2 \pm 7.8$ \\
\hline Body mass (kg) & $1.6 \pm 12.6$ & $-1.6 \pm 4.4$ & $-0.1 \pm 2.0$ \\
Body mass index (kg/m ${ }^{2}$ ) & $0.2 \pm 3.1$ & $-0.5 \pm 1.3$ & $-0.2 \pm 1.3$ & $-2.2 \pm 5.1$ \\
Waist circumference (cm) & $-0.8 \pm 6.7$ & $-3.5 \pm 4.3$ & $-2.2 \pm 4.1$ & $-2.2 \pm 17.8$ \\
Heart rate (bpm) & $1 \pm 2.5$ & $-2.6 \pm 12.5$ & $-4.5 \pm 10.6$ & $1.0 \pm 37.5$ \\
Systolic blood pressure (mm Hg) & $20 \pm 62.2$ & $-10.9 \pm 12.8$ & $-5 \pm 9.1$ & $-1 \pm 13.5$ \\
Diastolic blood pressure (mm Hg) & $5.5 \pm 19.9$ & $-4.0 \pm 9.0$ & $-4 \pm 7.4$ & $0.2 \pm 0.7$ \\
VO ${ }_{2}$ max (ml/kg/min) & $0.3 \pm 1.0$ & $0.2 \pm 0.7$ & $0.2 \pm 0.5$ & $1 \pm 2.8$ \\
Flexibility (cm) & $-0.3 \pm 2.5$ & $2.1 \pm 3.2$ & $1.1 \pm 2.4$ & $3.8 \pm 5.5$ \\
Abdominal resistance (reps) & $2.0 \pm 3.4$ & $5.4 \pm 6.6$ & $4 \pm 5.8 *$ & $3.0 \pm 5.5$ \\
Pectoral resistance (reps) & $0.2 \pm 4.7$ & $6.4 \pm 4.0$ & $2.5 \pm 6.2$ & $88.8 \pm 60.9$ \\
Leg press 1RM (kg) & $76.6 \pm 62.8$ & $100.8 \pm 5$ & $88.9 \pm 63.3$ & $8.5 \pm 10.6$ \\
Bench press 1RM (kg) & $9.3 \pm 13.2$ & $8.3 \pm 8.9$ & $8.1 \pm 10.3$ & $0.5 \pm 9.5$ \\
Blood glucose (mg/dl) & $-0.5 \pm 8.2$ & $-2.1 \pm 6.8$ & $3.7 \pm 11.9$ & $1.9 \pm 4.8$ \\
Insulin (mg/dl) & $2.1 \pm 6.2$ & $0.5 \pm 3.2$ & $3.1 \pm 4.8$ & $-5.4 \pm 36.1$ \\
Cholesterol (mg/dl) & $-26.2 \pm 27.9$ & $-1.1 \pm 42.3 *$ & $8.6 \pm 30.4$ & $-29.6 \pm 99.4$ \\
Triglycerides (mg/dl) & $-35.8 \pm 133.0$ & $-39.8 \pm 78.5$ & $-15.5 \pm 87.2$ & $7.3 \pm 36.8$ \\
HDL (mg/dl) & $22.0 \pm 63.6$ & $-0.4 \pm 6.6$ & $1.5 \pm 9.8$ & $-6.4 \pm 51.8$ \\
LDL (mg/dl) & $-33.1 \pm 43.6$ & $6.6 \pm 51.3$ & $5.2 \pm 53.3$ & $-3.1 \pm 34.1$ \\
VLDL (mg/dl) & $-15.0 \pm 39.4$ & $4.2 \pm 28.8$ & $0.5 \pm 33.3$ & $-18.4 \pm 378.4$ \\
Total lipids (mg/dl) & $-174.9 \pm 337.9$ & $79.5 \pm 339.8$ & $31.8 \pm 423.1$ &
\end{tabular}

* significant difference as compared with the control group $(p<0.05)$

CG - control group, NPTG - no personal trainer group, PTG - personal trainer group, 1RM - one-repetition maximum,

HDL - high-density lipoprotein, LDL - low-density lipoprotein, VLDL - very low-density lipoprotein 
mum on bench and leg press. In the NPTG, there was an improvement only in the sum of skinfolds. Therefore, the training showed that it might be enough to cause anthropometric, functional, and haemodynamic improvements since the CG remained with only significant changes in cholesterol. Data related to the comparison of the effect between groups are described in Table 4, which presents the deltas obtained with subtracting the post-training from the pre-training data.

As verified in Table 4, there are no significant differences between groups, except for abdominal resistance, which was higher in PTG in relation to CG (4 \pm 5.8 vs. $2.0 \pm 3.4 ; p<0.05$ ), and cholesterol, which was significantly lower in CG in relation to NPTG $(-1.1 \pm$ 42.3 vs. $-26.2 \pm 27.9 ; p<0.05)$.

\section{Discussion}

Our results indicate that ST performed with a personal trainer may develop more satisfactory results regarding anthropometric, haemodynamic, and functional parameters when compared with training performed without a personal trainer supervision. In our study, both groups performed the same type of ST. The group that underwent training with a personal trainer obtained a significant decrease in waist circumference, systolic blood pressure, skinfold, abdominal resistance, pectoral resistance, one-repetition maximum in supine and leg press. However, the group without a personal trainer had improvements only in the sum of the skinfolds.

Studies on ST showed positive effects on body composition, reducing the percentage of fat through an increase of the lean mass, which confirms our findings regarding the improvements in the sum of the skinfolds [10, 18]. According to some studies, ST performed in the medium to long term is associated with improvements in the body composition, since its practice implies an increase in metabolism due to an increase in muscle mass, energy expenditure, and bone mass, leading to cardiovascular adaptations.

It is estimated that the practice of ST is associated with a lower risk of developing metabolic diseases, with a positive effect on the levels of plasma lipids [19, 20]. After 8 weeks of ST, there were no changes in the lipid profile among the groups in our study. These results suggest that 8 weeks of ST with supervision or without supervision is not enough to induce changes in the lipid profile among sedentary subjects. It is clear that the duration and intensity of a training program are directly related to the degree of changes in the lipid profile. Acute effects of ST on parameters of lipo- protein metabolism were investigated, with significant increases in HDL and HDL3 and decreases in triglyceride levels after high-volume training. However, with low-volume training, no significant changes in plasma cholesterol, HDL, HDL2, HDL3, or triglycerides were reported [21]. Interestingly, in our study, the 8-week resistance training was enough to decrease systolic blood pressure in the PTG compared with NPTG. Accordingly, a study with strict personal trainer supervision of a 6-month combined aerobic and resistance training program reported decreased fasting insulin, highly sensitive C-reactive protein, leucocyte count, systolic high, and diastolic blood pressure [22].

This similar response between groups can be explained by the fact that, in the present study, there was no difference in total training volume. In this sense, one study [9] verified that progressive overload along with the total volume were fundamental components of ST and might contribute to the gain of strength and hypertrophy, factors responsible for several physiological adjustments. Thus, the present study suggests that the same volume during ST can generate similar results independently of the personalized follow-up.

Supervision of qualified personal trainers and the progression of intensity and volume of exercise are necessary to cause increases in physical fitness. The creation of stimuli in the exercise becomes effective as one begins to develop isolated sessions of training focused on the specific characteristics. Generating over-time progressions, variations, and overloads is required to achieve physiological adaptations and better performance. Individuals trained with the help of a personal trainer have greater strength gains than those who are not under supervision. A study that examined the impact of supervised and unsupervised ST in healthy older adults found that adherence to training equalled $92 \%$ in the supervised group and $97 \%$ in the unsupervised group, with improvements in 13 and 10 variables, respectively, which implies that supervised training was more effective in this population. A 12-week periodized heavy-resistance training directly supervised by a personal trainer vs. unsupervised demonstrated that supervised training resulted in a greater rate of training load increase and magnitude and maximal strength gains compared with unsupervised training [23, 24]. Our results showed improvements in abdominal and pectoral resistance, flexibility, leg press and bench press one-repetition maximum with only 8 -week periodized ST supervised by a personal trainer vs. unsupervised training. Another study with 12 weeks of ST in older adults observed more effective results in muscle power and balance 
A. Matos et al., The importance of a personal trainer in sedentary subjects

with supervised as compared with unsupervised training [23]. Thus, training accompanied by a professional can be a relevant factor in achieving performance results or in improving health.

The present study had some limitations that should be mentioned. First, although the volunteers were informed and agreed to the 8-week ST, some individuals did not complete $100 \%$ of the training protocol owing to other commitments; however, the frequency was carefully quantified in order to control the absence of some participants. The second limitation of the study consists in a limited number of volunteers, as well as the impossibility to determine the physiological mechanisms of the similar response between the PTG and NPTG regarding the same volume. The third limitation was the difference in age between the groups. This difference could have had an impact on some of the results, including the decrease in cholesterol levels in the CG as compared with NPTG. The concentration of some hormones, such as testosterone, depends on age and this could affect the interpretation of the program results. In addition, the lack of diet control and possible differences in occupational and leisure time activities could have influenced the outcomes. Finally, the large standard deviation in most of the variables is mainly explained by the small sample size in each group. In this perspective, future studies with a higher sample are suggested to elucidate the physiological effects of ST with and without a personal trainer.

Summing up, we showed that only 8 weeks of ST with a personal trainer could produce important changes in body composition and blood pressure among sedentary subjects. Therefore, the follow-up of a personal trainer is essential for precise adjustments in all variables to obtain satisfactory results through adequate and well-prepared training.

\section{Conclusions and practical applications}

It is understood as a practical application of the present study that ST is recommended as a non-pharmacological strategy for the senescent population owing to its peripheral effects such as strength gain, muscular endurance, reduction of peripheral vascular resistance, and decrease of the resting blood pressure, which could generate favourable metabolic adjustments to the maintenance of life. In this sense, the number of people looking for personalized services is increasing significantly. Thus, the present study offers data regarding the effect of ST with or without personal assistance on body composition, as well as haemodynamic and biochemical responses, suggesting the importance of understanding the total volume on the training result.

\section{Acknowledgments}

The study was supported by Fundação de Apoio a Pesquisa do Distrito Federal (FAPDF).

\section{Disclosure statement}

No author has any financial interest or received any financial benefit from this research.

\section{Conflict of interest}

The authors state no conflict of interest.

\section{References}

1. Guthold R, Stevens GA, Riley LM, Bull FC. Worldwide trends in insufficient physical activity from 2001 to 2016: a pooled analysis of 358 population-based surveys with 1.9 million participants. Lancet Glob Health. 2018;6(10):e1077-e1086; doi: 10.1016/S2214-109X(18) 30357-7.

2. Gorgui J, Gorshkov M, Khan N, Daskalopoulou SS. Hypertension as a risk factor for ischemic stroke in women. Can J Cardiol. 2014;30(7):774-782; doi: 10.1016/ j.cjca.2014.01.007.

3. Skrypnik D, Bogdański P, Mądry E, Karolkiewicz J, Ratajczak M, Kryściak J, et al. Effects of endurance and endurance strength training on body composition and physical capacity in women with abdominal obesity. Obes Facts. 2015;8(3):175-187; doi: 10.1159/000 431002.

4. Bakker EA, Lee D-C, Sui X, Artero EG, Ruiz JR, Eijsvogels TMH, et al. Association of resistance exercise, independent of and combined with aerobic exercise, with the incidence of metabolic syndrome. Mayo Clin Proc. 2017;92(8):1214-1222; doi: 10.1016/j.mayocp. 2017.02.018.

5. Strasser B, Schobersberger W. Evidence for resistance training as a treatment therapy in obesity. J Obes. 2011;2011:482564; doi: 10.1155/2011/482564.

6. Chupel MU, Direito F, Furtado GE, Minuzzi LG, Pedrosa FM, Colado JC, et al. Strength training decreases inflammation and increases cognition and physical fitness in older women with cognitive impairment. Front Physiol. 2017;8:377; doi: 10.3389/fphys.2017.00377.

7. Phillips MD, Patrizi RM, Cheek DJ, Wooten JS, Barbee JJ, Mitchell JB. Resistance training reduces subclinical inflammation in obese, postmenopausal women. Med Sci Sports Exerc. 2012;44(11):2099-2110; doi: 10.1249/MSS.0b013e3182644984.

8. Coutts AJ, Murphy AJ, Dascombe BJ. Effect of direct supervision of a strength coach on measures of muscular strength and power in young rugby league players. J Strength Cond Res. 2004;18(2):316-323; doi: 10.1519/ R-12972.1. 
9. Ratamess NA, Faigenbaum AD, Hoffman JR, Kang J. Self-selected resistance training intensity in healthy women: the influence of a personal trainer. J Strength Cond Res. 2008;22(1):103-111; doi: 10.1519/JSC. 0b013e31815f29cc.

10. Storer TW, Dolezal BA, Berenc MN, Timmins JE, Cooper CB. Effect of supervised, periodized exercise training vs. self-directed training on lean body mass and other fitness variables in health club members. J Strength Cond Res. 2014;28(7):1995-2006; doi: 10.1519/JSC.0000000000000331.

11. Dias MRC, Simão RF, Saavedra FJF, Ratamess NA. Influence of a personal trainer on self-selected loading during resistance exercise. J Strength Cond Res. 2017; 31(7):1925-1930; doi:10.1519/JSC.0000000000001663.

12. Jackson AS, Pollock ML. Generalized equations for predicting body density of men. Br J Nutr. 1978;40(3): 497-504; doi: 10.1079/bjn19780152.

13. Jackson AS, Pollock ML. Generalized equations for predicting body density of men. 1978. Br J Nutr. 2004; 91(1):161-168.

14. Baltaci G, Un N, Tunay V, Besler A, Gerçeker S. Comparison of three different sit and reach tests for measurement of hamstring flexibility in female university students. Br J Sports Med. 2003;37(1):59-61; doi: 10.1136/bjsm.37.1.59.

15. Alaranta H, Hurri H, Heliövaara M, Soukka A, Harju R. Non-dynamometric trunk performance tests: reliability and normative data. Scand J Rehabil Med. 1994;26(4): 211-215.

16. Mayhew JL, Ball TE, Arnold MD, Bowen JC. Pushups as a measure of upper body strength. J Strength Cond Res. 1991;5(1):16-21.

17. Bangsbo J, Iaia FM, Krustrup P. The yo-yo intermittent recovery test: a useful tool for evaluation of physical performance in intermittent sports. Sports Med. 2008;38(1):37-51; doi: 10.2165/00007256-200838010 -00004 .

18. Miller T, Mull S, Aragon AA, Krieger J, Schoenfeld BJ. Resistance training combined with diet decreases body fat while preserving lean mass independent of resting metabolic rate: a randomized trial. Int $\mathrm{J}$ Sport Nutr Exerc Metab. 2018;28(1):46-54; doi: 10.1123/ ijsnem.2017-0221.

19. Da Silva JL, Vinagre CGCM, Morikawa AT, Alves MJNN, Mesquita $\mathrm{CH}$, Maranhão RC. Resistance training changes LDL metabolism in normolipidemic subjects: a study with a nanoemulsion mimetic of LDL. Atherosclerosis. 2011;219(2):532-537; doi: 10.1016/j.atherosclerosis.2011.08.014.

20. Prabhakaran B, Dowling EA, Branch JD, Swain DP, Leutholtz BC. Effect of 14 weeks of resistance training on lipid profile and body fat percentage in premenopausal women. Br J Sports Med. 1999;33(3):190-195; doi: 10.1136/bjsm.33.3.190.

21. Wallace MB, Moffatt RJ, Haymes EM, Green NR. Acute effects of resistance exercise on parameters of lipopro- tein metabolism. Med Sci Sports Exerc. 1991;23(2): 199-204.

22. Stefanov T, Vekova A, Bonova I, Tzvetkov S, Kurktschiev D, Blüher M, et al. Effects of supervised vs nonsupervised combined aerobic and resistance exercise programme on cardiometabolic risk factors. Cent Eur J Public Health. 2013;21(1):8-16; doi: 10.21101/cejph.a3801.

23. Lacroix A, Kressig RW, Muehlbauer T, Gschwind YJ, Pfenninger B, Bruegger O, et al. Effects of a supervised versus an unsupervised combined balance and strength training program on balance and muscle power in healthy older adults: a randomized controlled trial. Gerontology. 2016;62(3):275-288; doi: 10.1159/0004 42087.

24. Mazzetti SA, Kraemer WJ, Volek JS, Duncan ND, Ratamess NA, Gómez AL, et al. The influence of direct supervision of resistance training on strength performance. Med Sci Sports Exerc. 2000;32(6):1175-1184; doi: 10.1097/00005768-200006000-00023. 